\title{
Vehicle Detector Method for Complex Environments on Embedded Linux Platform
}

\author{
A.Shanthi \\ M.Tech Student \\ DECS Department of ECE \\ Vardhaman College of Engineering \\ Shamshabad, Hyderabad, Andhra Pradesh, India
}

\author{
M.P.Sirisha \\ Assistant Professor \\ Department of ECE \\ Vardhaman College of Engineering \\ Shamshabad, Hyderabad, Andhra Pradesh, India
}

\begin{abstract}
Traffic surveillance and investigation is important to increase the safety on roads. Vehicle detection and tracking in road surveillance is classical task in computer vision and critical component in modern traffic monitoring system. Intelligent transport system (ITS) brings advanced computing, sensing and telecommunication technologies to transport related problems. Many traffic surveillance systems have been developed to detect traffic congestion. In this paper video traffic detection system is used to detect the vehicles and track the vehicle speed. This project is implemented using Open CV Image processing library. In implementing this project gray scale conversion, background subtraction, and morphological operations are used. This proposed system uses a webcam located in traffic surveillance system. It captures video stream and send to ARM9 microcontroller; it processes the information such as number of vehicles, vehicle speed and send to remote PC.
\end{abstract}

\section{Keywords}

ITS, Grayscale, Open CV, Background Subtraction Morphological Operations.

\section{INTRODUCTION}

The traffic information is important in maintenance of modern transportation system. Traffic surveillance technologies play vital role in incident detection and traffic management. Traffic monitoring based on vision system in huddled areas is difficult to detect vehicles. Adaptive vehicle detector approach for complex environments [1] detects vehicles in complex environments and provides methods for vehicle tracking. This system uses histogram extension, gray level differential value methods are used to detect moving objects.

Video based traffic monitoring system [2] is more advantageous compared to other methods. In addition to vehicle counting more traffic information can be obtained by images. Video cameras can be easily installed and used in mobile environments. Real time vehicle detection in video stream relies heavily on image processing techniques, such as motion detection, edge detection and digital filtering etc. Different vision based detection techniques are proposed by researchers [3-6] like wavelet based tracking, AVG vision which used Hough transform, background segmentation, object tracking ,adaptive thresholding techniques were used. Joint random filed method [7] proposed method for vehicle detection developed algorithm for real time vehicle detection for video based traffic monitoring but unable to find speed of vehicles. Real time incremental segmentation and tracking of vehicles [8] this system mounted a camera relatively low to ground to avoid spill over effects, tracks vehicles at low camera angles using stable features. An enhanced back ground estimation algorithm [9] proposed background subtraction using sigma delta filter.

A real time computer vision system for vehicle tracking and traffic surveillance proposed method for detecting vehicles in complex environments using features based techniques. There were many techniques for vision based systems to detect vehicles but they were developed using MATLAB. MATLAB is slow to process the videos. Another drawback of MATLAB is that it can work only with recorded videos.

The proposed system use Linux platform (ubuntu), and Open $\mathrm{CV}$ image processing library which is fast in processing in videos than MATLAB.ARM9 microcontroller processes the information. The information is displayed on ARM9 board and information is sent to remote PC.

\section{PROPOSED METHOD}

The proposed system shown in figure1, use vision based system to monitor the traffic. The traffic is monitored by webcam located at high spot. The application of computer vision techniques to analyse video provides improvement over existing methods of traffic monitoring systems. The proposed system is implementedt on ARM9 S3C2440 mini board. The ARM9 processor detects the vehicles, counts and calculates the speed of each vehicle.

\section{FUNCTIONAL REQUIREMENTS}

2.1 Capturing the Video from Traffic Scene The first stage of the project, the videos are captured using a fixed camera. Video cameras are standard equipment for modern transportation and management. Traffic surveillance is important to update the information about the movement of traffic. The camera is located at high spot as shown in figure2, it is positioned in such a way that vehicles are coming towards the camera in order to avoid the side views of movement of vehicles and overlapping of vehicles.

\subsection{Extracting Frames from Video}

A video sequence is a series of still images with a small interval between two images. The video sequence is converted to frames. OpenCV image processing library convert the video which is in AVI format into frames. It captures the video stream and stores the frames in the buffer. 


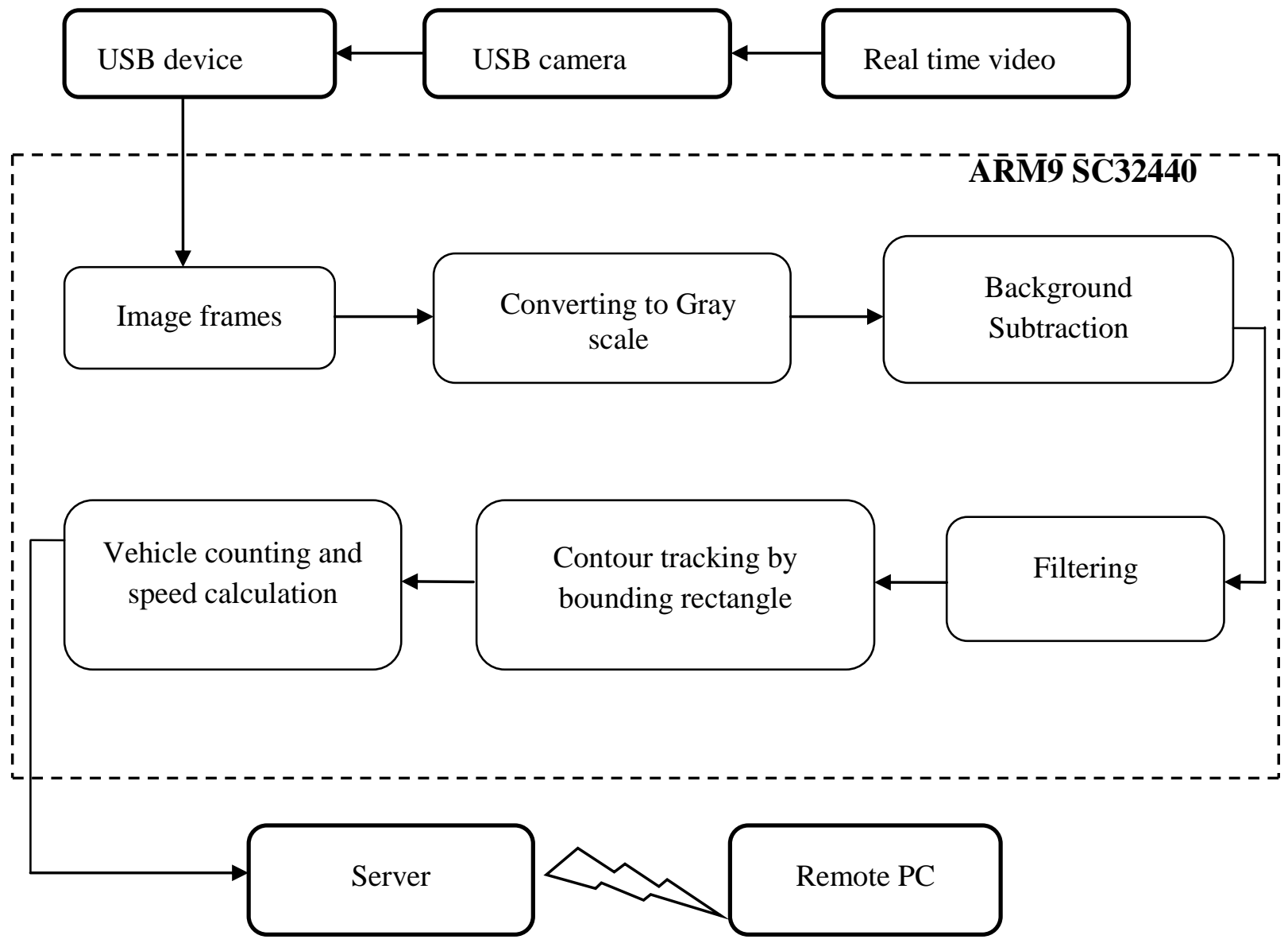

Figure: 1 Implementation of the Proposed System

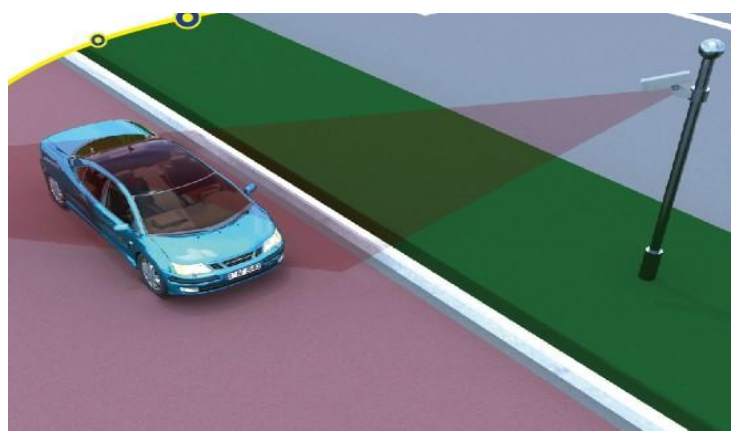

Figure 2: Capturing Video from Camera

\section{3 .Gray scale Conversion}

The video which is converted to frames are still color images as shown in figure3, which contain RGB values. To remove noise from the images filtering techniques are applied. To apply filtering techniques the image should be gray scale image. Gray scaling converts color image to gray scale by removing color values as shown in figure4. Thus complexity in processing image can be reduced compared to color image

\subsection{Background Subtraction}

In the video of traffic scene on the road there are moving vehicles and stationery objects like trees, buildings. In detecting the moving objects a scene contains the stationary objects called background frame and the moving objects such as vehicles called foreground frame. Background subtraction is the common techniques to detect the moving vehicles. In this process video frame is used to build the background model by calculating the values of each pixel in an input image. Background subtraction method separates every pixel of each frame into background and foreground region. Background subtraction technique is widely used to detect moving vehicles. It subtract background image from the input image frame to detect the vehicles in the foreground as shown in figure6.

\subsection{Filtering}

Morphological operations are set of operations that process based on shapes. Morphological operations apply structuring element to an input image and generate output image. Basic morphological operations are Dilation and Erosion. It is filtering technique to detect vehicles, remove noise, isolation of individual elements and joining disparate elements in an image.

Dilation operation thickens objects in an image in a specific manner and thickness is controlled by structuring element. Dilation operation compares centre pixel with the neighborhood pixel. If any neighborhood pixels are foreground pixel then the background pixel is switched to 
foreground pixel. The structuring element is scanned over the image, and computes maximum pixel value overlapped by the structuring element and replaces the image pixel in structuring element position with maximum value. This maximizing operation cause bright regions to grow as shown in figure7. Erosion operation is opposite to dilation it thins objects in an image in a specific manner and thinness is controlled by structuring element. The structuring element is scanned over the image and computes minimum pixel value overlapped over the structuring element and replaces the image pixel in structuring element position with minimum value. This minimizing operation cause bright areas of the image get thinner where as dark zones gets bigger as shown in figure8.

\subsection{Contour}

Contour tracking tracks the moving vehicles. Moving vehicles are tracked by bounding contour and keep updating continuously.

\subsection{Vehicle counting and speed calculation}

Vehicles are counted within the region of interest. When vehicles enter into the ROI (region of interest) the system identifies the vehicle by creating bounding box to that vehicle. Thus the vehicles are counted in that particular region. When vehicle moves from one frame to other frame the speed of the vehicle is calculated as shown in figure9.

\section{IMPLEMENTATION}

Video of the traffic on the road is captured using the camera. The video information is regarded as the input to the whole system. OpenCV functions are used to capture and retrieve the video. The following code is used to capture and retrieve the video stream.

CvCapture $*$ Capture $=0$;

IpIImage $*$ frame $=0$;

CvCapture $*$ Capture $=$ CvCaptureFromCAM(CV_CAP_ANY $)$;

Frame $=$ cvRetrieveFrame capture (capture);

CvReleaseCapture(\&capture);

Opencv library includes JPG decompression and convert it into IpIImage format. The frames are extracted to image format.

IpIImage $*$ input image $=\mathrm{CvQuery} F$ rame (capture);

In Processing the image the main problem of detecting vehicles is that it contains noise. The quality of image may be poor because of the lightening conditions, noise etc. The noise can be removed by using filtering techniques such as dilation and erosion. Thus vehicles are detected, counted and the speed of each vehicle is calculated using the OpenCV techniques.

\subsection{System Requirements}

Operating System: Linux (ubuntu 12.04)

Image Processing Library: OpenCV

GUI Library: wxWidgets

Cross Platform: Qt

Development Board: Mini2440 S3C2440 ARM9 board

\subsection{Working Principle}

The embedded system is designed to detect vehicles in complex environments. The camera is interfaced to the ARM microcontroller to detect the vehicles. Camera is used to capture video stream continuously. The ARM microcontroller processes the video streams and detect the number of vehicles and speed of the each vehicle is calculated as shown in figure 13.The proposed system makes use of embedded board which consumes less power and advanced micro controller like
S3C2440. S3C2440 is a Samsung's microcontroller which is designed based on structure of ARM20T family. This works for a voltage of $+3.3 \mathrm{v}$ and operating frequency $400 \mathrm{MHz}$. S3C2440 comes with Friendly ARM board or mini 2440 board. In order to work with ARM9 micro controller three things are required:

1. Boot loader

2. Kernel

\section{Root file system}

Boot loader is to initialize all the devices that are present on the mother board mini 2400 and also find out any problem in devices of the motherboard mini 2440.Boot loader finds different operating systems present in storage device and display it on the screen. It also loads operating system related files in to temporary memory RAM.

This proposed system uses super vivi boot loader which is specific to mini 2440 board. Kernel is the core part of the operating system. This proposed system use kernel of embedded Linux. When embedded Linux is loaded the device related drivers that are present on the mother board are automatically loaded. Root file system is a file system tells how files are arranged in the internal storage device. In embedded Linux, kernel treats everything as file even input and output devices also.

The essential programs required for MINI 2440 are, boot loader, embedded Linux kernel, root file system is loaded in NOR flash which is present on the MINI2440 board itself. The programs related to applications are loaded in NAND flash it is also present on board itself. By using boot strap switch present on the board will help user to select NAND or NOR flash. Once loading everything into MINI 2400 board it starts working based on application program loaded in NAND flash.

The camera is interfaced to the mini board, captures the video stream of vehicles in that particular location. The process of video capture is shown in figure2.To detect the motion of vehicles, first open video device, capture video stream from the camera and grab the frame from the video. Then read images from the grabbed frame and store the image in particular memory location. Then processor read already stored images, current image and already stored images are converted to gray scale image. Then compare both the images and differentiate them. Contour to the image is found by drawing bounding rectangles so that moving vehicles are tracked in the particular region of interest.

The controller draws two regions. The number of rectangles present in first region shows number of vehicles present in that particular region. Controller tracks the vehicles and calculated how many frames rectangles move from one region to other gives the speed of the vehicle. Thus number of vehicles in particular regions is detected and speed of each vehicle is calculated. This information is displayed on the S3C2440 ARM9 board and information is sent to remote PC. 


\subsection{Experimental Results}

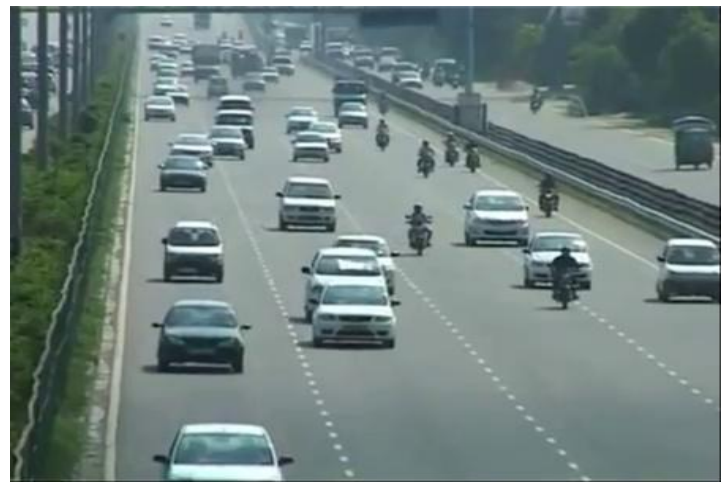

Figure 3: RGB Source Image

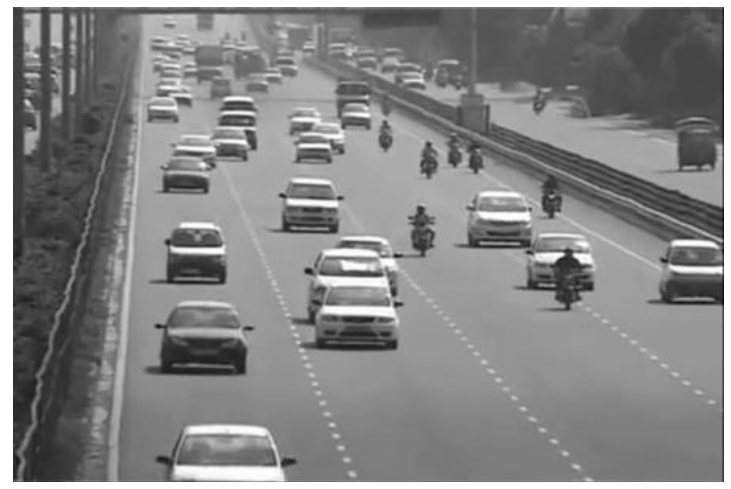

Figure 4: Result of Gray scale Image

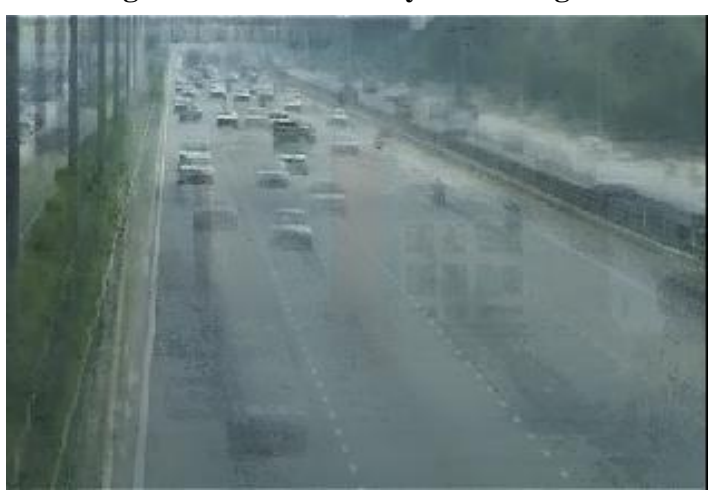

Figure 5: Result of Background Extracted from the Source image

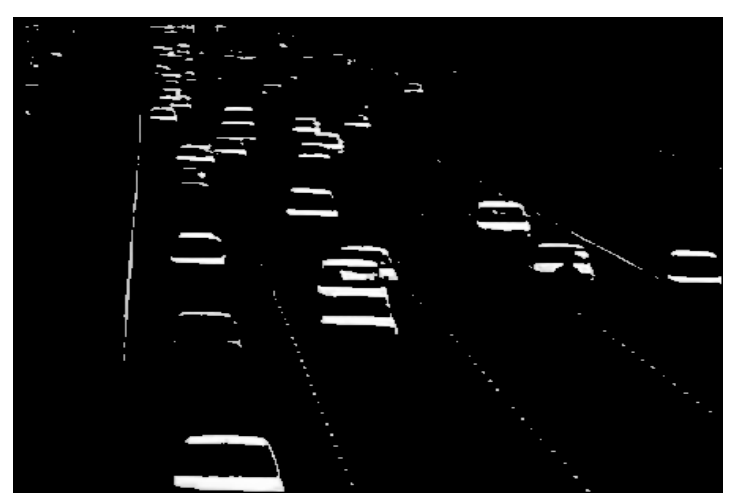

Figure 6: Result of Foreground Image

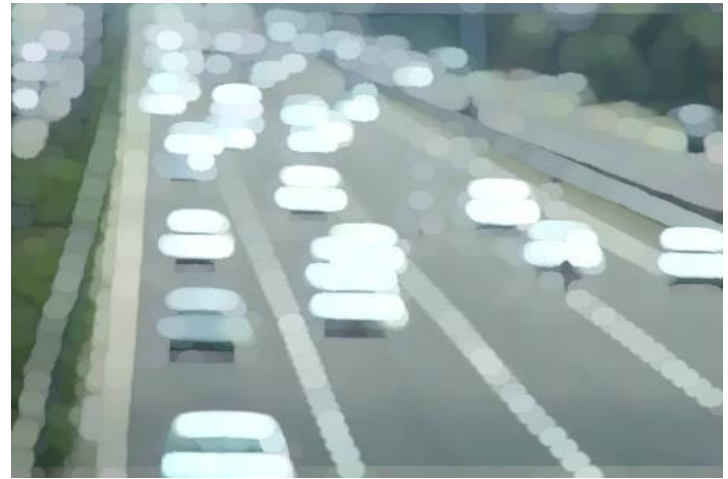

Figure 7: Result of Dilation Image

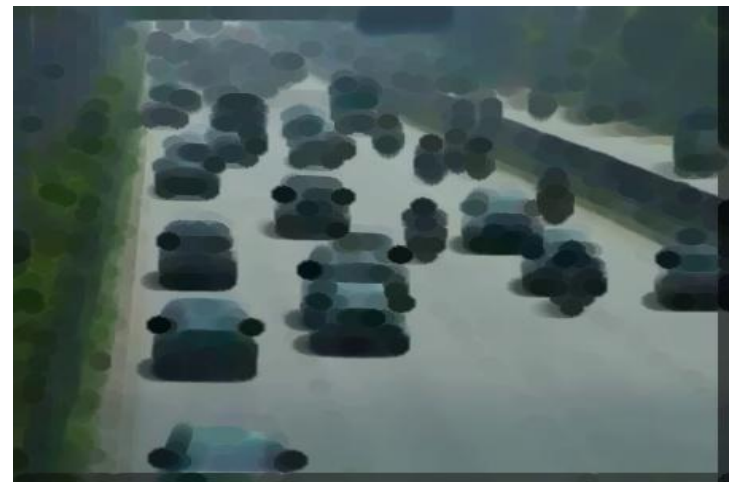

Figure 8: Result of Erosion Image

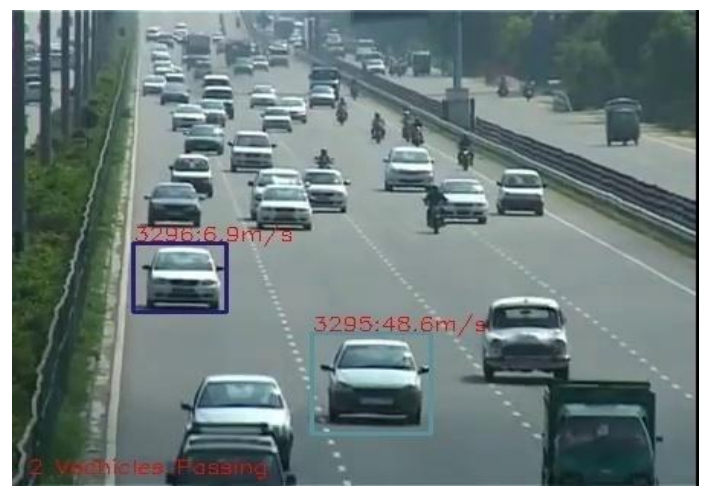

Figure 9: Result of Daytime Image vehicle count and speed calculation

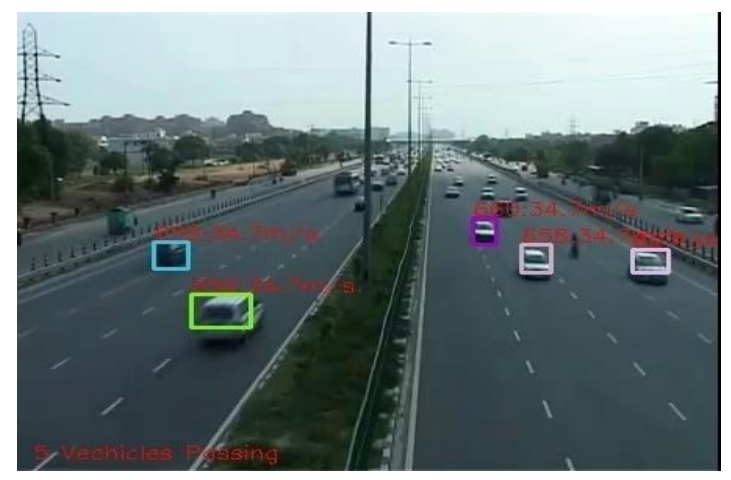

Figure 10: Result of Evening Image vehicle count and speed calculation 


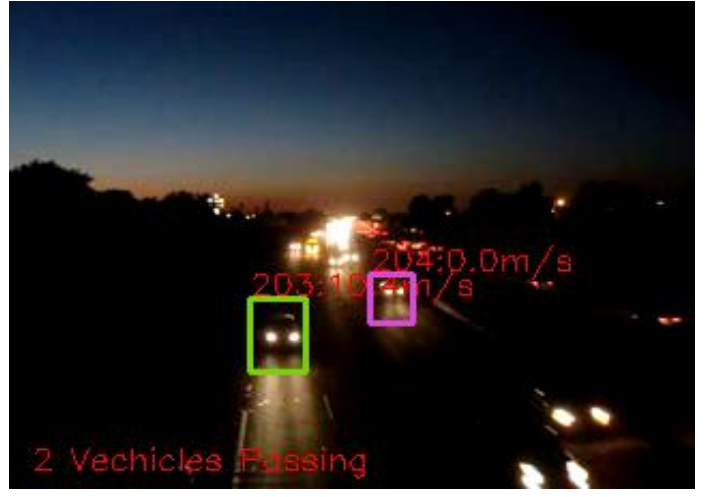

Figure 11: Result of Night Image vehicle count and speed calculation

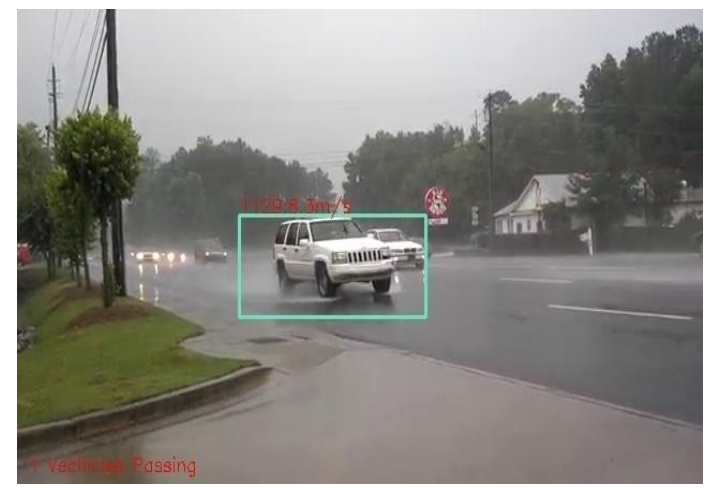

Figure 12: Result of Rainy Image vehicle count and speed calculation

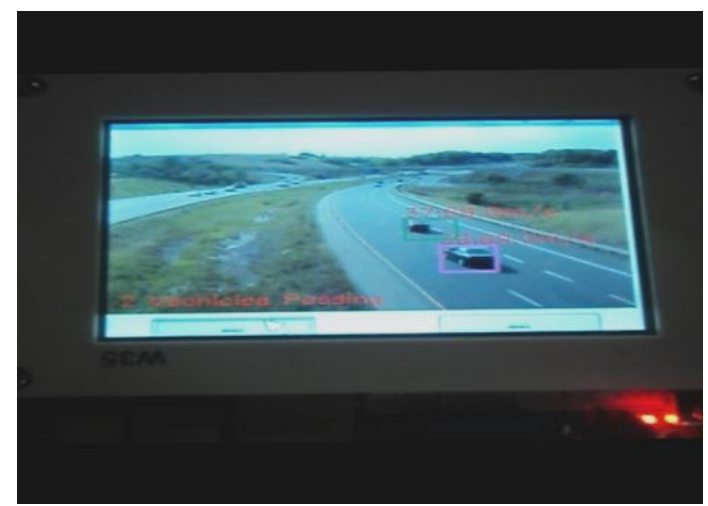

Figure 13: Experiment result on ARM9 Board S3C2440

\section{CONCLUSIONS}

This paper presents traffic monitoring approach for vehicle tracking, counting and speed of vehicles using OpenCV image processing library. This project is developed by integrating all hardware components and software. OpenCV image processing library is used to extract the vehicle details from the background. Background subtraction and filtering techniques are applied to track the vehicles. The contour based tracking by bounding rectangles is used to count the vehicles in region of interest. This embedded project uses ARM9 to process the video. Every module is placed carefully thus contributing to the best working of the system. Using highly advanced ARM9 board and with the help of growing technology the project is implemented successfully.

\section{REFERENCES}

[1] "Adaptive Vehicle Detector Approach for Complex Environments" Bing-Fei Wu ; Inst. of Electr. \& Control Eng., Nat. Chiao Tung Univ., Hsinchu, Taiwan ; JhyHong Juang Intelligent Transportation Systems, IEEE Transactions on (Volume:13, Issue: 2 )

[2] "A video-based real-time vehicle detection method by classed background learning" Xiaoju Tan et al, World Transactions on Engineering and Technology Education Vol.6, No.1, 2007

[3] "Wavelet-based vehicle tracking for automatic traffic surveillance" Kim, J.B. ;Comput. Eng. Dept., Kyungpook Nat. Univ., Taegu, South Korea ; Lee, C.W. ; Lee, K.M. ; TENCON 2001. Proceedings of IEEE Region 10 International Conference on Electrical and Electronic Technology (Volume:1 )

[4] "A study on recognition of lane and movement of vehicles for port AGV vision system" Jin Woo Lee ; Dept. of Electr. Eng., Univ. of Dong-A, Busan ; Jung Ho Kim ; Young Jin Lee ; Kwon Soon Lee. Industrial Electronics, 2002. ISIE 2002. Proceedings of the 2002 IEEE International Symposium on (Volume:2 )

[5] "Spatiotemporal vehicle tracking: the use of unsupervised learning-based segmentation and object tracking" Shu-Ching Chen ; Sch. of Comput. Sci., Florida Int. Univ., Miami, FL, USA ; Mei-Ling Shyu ; Peeta, S. ; Chengcui Zhang . Robotics \& Automation Magazine, IEEE (Volume:12, Issue: 1 )

[6] Wang, Y.K. and S.H. Chen. "Robust Vehicle Detection Approach" Advanced Video and Signal Based Surveillance, 2005. AVSS 2005. IEEE Conference

[7] Y.Wang "Joint Random Field Model for All Weather Moving Vehicle Detection.”IEEE trans. image process. Vol 19 no 9 sep 2010

[8] N.K.Kanhere et al, "Real-time Incremental Segmentation and Tracking of Vehicles at Low Camera Angels Using Stable Features." IEEE Trans intell transp.syst. vol 9 no1 mar 2008

[9] M.Vargas, et al, "An Enhanced Background Estimation Algorithm For Vehicle Detection In Urban Traffic Scenec.'IEEE trans. Veh .technol.. vol 59 no 8 oct 2010

[10] Benjamin Coifman, David Beymer, Philip McLauchlan Jitendra Malik "A real-time computer vision system for vehicle tracking and traffic surveillance."

[11] Learning OpenCV by Gary Bradski and Adrian Kaehler. 